\title{
Update of S-NPP VIIRS Thermal Emissive Bands Radiometric Calibration Stability Monitoring Using the Moon
}

\author{
Zhipeng Wang*a, Xiaoxiong Xiong ${ }^{\mathrm{b}}$, and Yonghong $\mathrm{Li}^{\mathrm{a}}$ \\ ${ }^{a}$ Science Systems and Applications, Inc., 10210 Greenbelt Rd, Lanham, MD 20706 \\ ${ }^{\mathrm{b}}$ Sciences and Exploration Directorate, NASA/GSFC, Greenbelt, MD 20771
}

\begin{abstract}
The Suomi-NPP VIIRS thermal emissive bands (TEB) are radiometrically calibrated on-orbit with reference to an onboard blackbody (BB) regularly operated at approximately $292.5 \mathrm{~K}$. The calibration stability at other temperature ranges can be evaluated based on the observations of remote targets with stable thermal properties, such as the Moon. VIIRS has scheduled viewings of the Moon on a nearly monthly basis at a phase angle of nearly -51 degrees. In this study, the brightness temperatures (BT) of the lunar surface retrieved using the detector gain coefficients calibrated with the BB are trended to monitor the calibration stability of VIIRS TEB. Since the Lunar surface temperatures are spatially non-uniform and vary greatly with the photometric geometry, the BT trending must be based on the same regions of the Moon under the same solar illumination condition. Also, the TEB lunar images are always partially saturated because the highest lunar surface temperatures are beyond the dynamic range of all VIIRS TEB detectors. Therefore, a temporally invariant dynamic mask is designed to clip a fraction of the lunar images corresponding to the regions of the Moon that may saturate the detector at any lunar event. The BT of the remaining hottest pixels are then trended. Results show that, since the launch of VIIRS to mid-2016, the radiometric calibration of all TEB detectors has been stable within $\pm 0.4 \mathrm{~K}$ at the $\mathrm{BT}$ range of as high as $350 \mathrm{~K}$.
\end{abstract}

Keywords: VIIRS, Moon, thermal emissive bands (TEB), radiometric calibration

\section{INTRODUCTION}

The Visible Infrared Imaging Radiometer Suite (VIIRS) is a passive whisk-broom scanning imaging spectroradiometer aboard Suomi National Polar-orbiting Partnership (NPP) satellite that was launched on October, $28^{\text {th }}, 2011^{1}$. It operates on a Sun-synchronous polar orbit and provides high quality radiometric calibrated data globally. VIIRS has a rotating telescope assembly (RTA) that can image a swath of $3000 \mathrm{~km}$ in the along-scan direction (cross-track) and $12 \mathrm{~km}$ in the along-track direction at nadir in each scan. A subsequent double-sided half angle mirror (HAM) rotates at half the speed of the RTA to de-rotate the image.

VIIRS has 22 spectral bands including 14 reflective solar bands (RSB), 1 panchromatic day/night band and 7 thermal emissive bands (TEB). Covering a spectral range from 3.7 to $12.2 \mu \mathrm{m}$, these TEBs measure the thermal emission day and night at a nominal $375 \mathrm{~m}$ nadir resolution for the imaging ( $\mathrm{or}$ ' $\mathrm{I}$ ') bands I4-I5 and $750 \mathrm{~m}$ for the moderate resolution (or ' $\mathrm{M}$ ') bands M12-M16, The key design specifications of the TEBs are listed in Table 1. Among the TEB, band M13 is a dual gain (DG) band that uses two detector gain stages to cover wider dynamic range. Other TEBs have single gain (SG) setting. According to their center wavelengths, the VIIRS spectral bands are located on three separate focal plane assemblies (FPA): visible/near-infrared (VIS/NIR), short-wave and mid-wave infrared (S/MWIR), and long-wave infrared (LWIR). These spectral bands are spread in the along-scan direction while the detectors within a band are assembled in the along-track direction. All the TEBs are located on the S/MWIR and LWIR FPAs, which are often referred to as the cold focal plane assemblies (CFPA) because these two FPAs are cryogenically cooled by a passive radiative cooler to operate at a nominal temperature of $\sim 82.5 \mathrm{~K}$.

Similar to its predecessor the MODerate resolution Imaging Spectroradiometer (MODIS) currently operating aboard NASA's Terra and Aqua satellites, the VIIRS instrument is radiometrically calibrated by a set of onboard calibrators $(\mathrm{OBC})$ including a solar diffuser, a solar diffuser stability monitor and a blackbody $(\mathrm{BB})^{2}$. The BB is the primary source for TEB calibration. During its regular operation, the BB temperature is well controlled at a nominal temperature of 292.5 K monitored by 6 embedded thermistors. The radiance from BB can be calculated based on Planck's equation. The detector responses when viewing deep space through the space view (SV) port provides the background signal. After 
compensating for the background emission, the radiance at detector $\Delta L_{\text {det }}$ and the background corrected detector response in digital number $d n$ are known for the BB view. A quadratic algorithm is used to relate $d n$ to $\Delta L_{d e t}{ }^{3,4}$

$$
\Delta L_{\mathrm{det}}=F \cdot\left(c_{0}+c_{1} \cdot d n+c_{2} \cdot d n^{2}\right),
$$

where $c_{0}, c_{1}$ and $c_{2}$ are the detector gain coefficients calibrated pre-launch. The F-factor $F$ is the gain correction factor. For each pixel, the radiance is calculated and then the brightness temperature (BT) is retrieved from the radiance, after the correction of background radiance, through Planck's equation. The primary on-orbit radiometric calibration task for TEB is to determine the F-factor on a scan-by-scan basis at regular operation. The BB warm-up cool-down (WUCD) operation is executed every quarter to evaluate the non-linear coefficients $c_{0}$ and $c_{2}$. During the operation, the BB temperature is heated to $315 \mathrm{~K}$ first and then cooled down to the ambient temperature of $\sim 267 \mathrm{~K}$. The $\Delta L_{d e t}$ and the $d n$ at different temperatures are quadratically fitted to derive $c_{0}, c_{1}$ and $c_{2}$ using Eqn. (1). The results are evaluated by comparing with the pre-launch values. Since launch, $c_{0}, c_{1}$ and $c_{2}$ have been stable and have not been updated ${ }^{5}$.

Table 1. VIIRS TEB design specification ${ }^{1}$.

\begin{tabular}{|c|c|c|c|c|c|c|}
\hline Band & $\mathbf{C W}(\mu \mathrm{m})$ & Gain Stage & Sampling Interval $(\mathbf{m})$ & $\mathbf{T}_{\text {typ }}(\mathbf{K})$ & $\mathbf{T}_{\max }(\mathbf{K})$ & NEdT $(\mathbf{K})$ \\
\hline M12 & 3.700 & Single & $0.742 X 0.776$ & 270 & 353 & 0.396 \\
\hline M13 & 4.050 & High/Low & $0.742 X 0.259$ & $300 / 380^{*}$ & $343 / 634^{*}$ & $0.107 / 0.423^{*}$ \\
\hline M14 & 8.550 & Single & $0.742 X 0.776$ & 270 & 336 & 0.091 \\
\hline M15 & 10.763 & Single & $0.742 X 0.776$ & 300 & 343 & 0.07 \\
\hline M16 & 12.013 & Single & $0.742 X 0.776$ & 300 & 340 & 0.072 \\
\hline I4 & 3.740 & Single & $0.371 X 0.387$ & 270 & 353 & 2.5 \\
\hline I5 & 11.450 & Single & $0.371 X 0.387$ & 210 & 340 & 1.5 \\
\hline$*:$ M13 has high-gain and low-gain stages with different $\mathrm{T}_{\text {typ }}, \mathrm{T}_{\max }$ and NEdT (noise equivalent temperature) \\
\hline
\end{tabular}

Although the on-orbit calibration of the TEB primarily relies on the on-board BB, observations over well-characterized remote targets have been used by VIIRS Characterization Support Team (VCST) analysts for post-launch validation purpose. Among these targets, the Moon has become a more and more important target because of its spatial, spectral, and radiometric stability in the long-run ${ }^{6}$. It has been used by MODIS Characterization Support Team (MCST) to assess the calibration accuracy of MODIS TEB ${ }^{7}$. This paper summarizes our recent efforts to study the long-term stability of the TEB using lunar surfaces as the reference. Following the TEB calibration overview, Section 2 will briefly introduce the VIIRS lunar observation and the radiometric property of lunar surfaces. Section 3 will provide the methodologies used in our study. The results and further discussion are given in Section 4. Section 5 is the summary.

\section{LUNAR OBSERVATION OF VIIRS THERMAL EMISSIVE BANDS}

\subsection{VIIRS Lunar observation}

The Moon has been widely accepted as a stable source for on-orbit calibration of remote sensing instruments. Regular VIIRS lunar observations are scheduled on a nearly monthly basis at a phase angle of nearly -51 degrees (waxing gibbous $)^{8}$. The lunar irradiance strongly depends on the photometric factors such as lunar phase particularly. To minimize the brightness variation of the Moon among events to reduce the calibration uncertainty, the lunar phases at the time of calibration are mostly limited within a small range of \pm 0.5 degrees. The criterion was loosened to allow a lunar phase angle beyond this range for only a few events or the observation cannot be made for those months.

VIIRS observes the Moon through its space view (SV) port at an elevation angle of 24.325 degrees below the instrument $\mathrm{X}-\mathrm{Y}$ plane. Limited by the orbit of the satellite and the Moon, a satellite roll maneuver around its $\mathrm{X}$ axis, or the moving direction, is usually required to ensure the Moon passes the center of the field of view of the SV port. One lunar calibration event takes less than five minutes to complete. The VIIRS HAM scans at a period of 1.78 seconds, so multiple scans of lunar images can be acquired during one calibration event. For one event, the Moon enters the field-ofview (FOV) of the FPA from the top ( $+\mathrm{X}$ direction in VIIRS coordinate), then moves downward across the detector array and exits the FOV at the bottom (-X direction). Electric sector rotation is applied during the lunar calibration so the Moon image captured by the SV port is read out from the Earth view data sector. 
The diurnal period of the Moon is equal to the synodic period, so observers at the Earth almost always view the same side of the Moon, subject to the libration effect. The lunar images acquired by VIIRS during different scheduled lunar calibrations have similar shapes since the lunar phases are also similar. A few examples are shown in Fig. 1: the lunar images of VIIRS band I1 at $0.64 \mu \mathrm{m}$ and two thermal bands I4 and M12, acquired at a scan during four separate events in 2013. In the plot, the horizontal direction is the along-scan direction and the vertical direction is the along-track direction. I-band images are composed 32 rows of detectors/pixels and 32 columns of image frames/pixels. M-band images are composed of 16 rows of detectors/pixels and 16 columns of image frames/pixels.
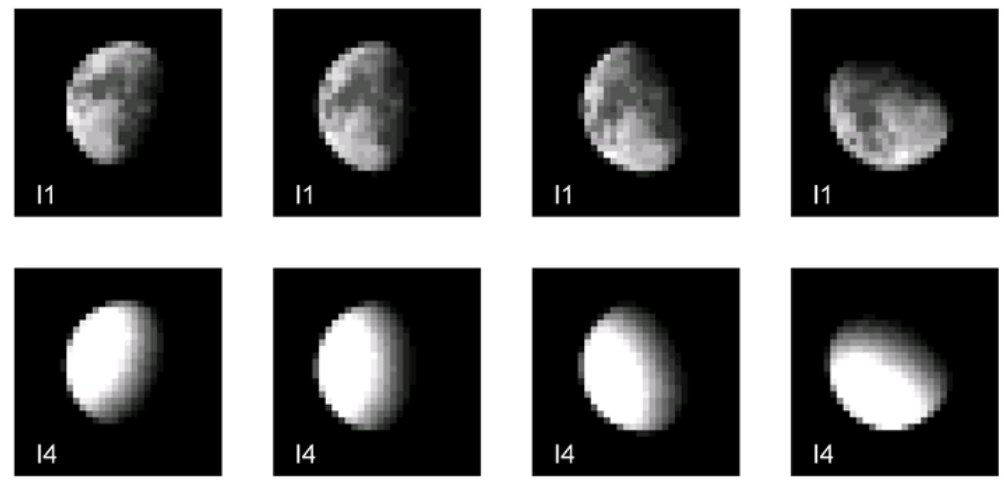

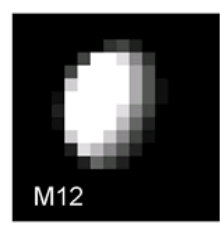

2013_03_23

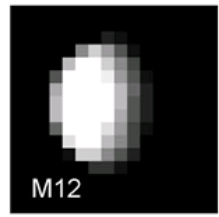

2013_04_21

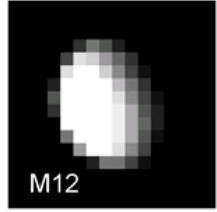

2013_05_21

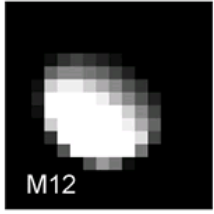

2013_10_14

Figure 1. Lunar images of a few VIIRS bands captured at the middle of various lunar calibration events.

By design, the images of different bands are spatially co-registered to cover the same scene. The orientation of the lunar images vary among events, depending on the moving direction of the satellite in reference to the moving track of the Moon. This, together with the fact that the distance between the Moon and the satellite varies, means that the lunar images of different events are not spatially co-registered. The orientations of the rotation can be quantified by the illumination angle of the Sun in reference to the SV pointing direction ${ }^{9}$. Similar to MODIS, the ground instantaneous FOV (IFOV) of VIIRS detectors on the Moon is rather large $(173 \mathrm{~km} \times 173 \mathrm{~km}$ for I bands and $346 \mathrm{~km} \times 346 \mathrm{~km}$ for M bands). The spatial variation of the lunar surface within a pixel is rather significant. This implies that even if the images from various events can be projected into selenographic coordinates, the pixels of different events will not be properly co-registered due to rotation.

\subsection{Thermal properties of the lunar surfaces}

The Moon has no internal heat source so the heating comes almost entirely from the incident solar radiation. Its surface is made up almost entirely of rocky materials with low thermal conductivity and relatively low heat capacity. As a result, the surface temperature quickly reaches equilibrium with incoming solar radiation during the lunar daytime. The StefanBoltzmann law can be used to estimate the planet surface temperature at thermal equilibrium

$$
I=\varepsilon \sigma T^{4},
$$

where $I$ is the radiated energy per unit area, which equals to the absorbed solar energy per unit area. $T$ is the absolute surface temperature in units of Kelvin, $\varepsilon$ is the emissivity, and $\sigma$ is Stefan's constant. For a lunar surface with the Sun directly overhead, such as the horizontal region near the equator at lunar noon, $I$ is close to the solar constant $1368 \mathrm{~W} / \mathrm{m}^{2}$ considering the Moon is at Earth's neighborhood, minus the portion reflected. Since the emissivity is close to 1 minus the reflectance, those two terms cancel out. When the Sun is not directly overhead, the surface temperature will be lower due to the oblique illumination. Because the lunar spinning axis is tilted only 1.54 degrees from the ecliptic, solar angles at noon are always within 1.5 degrees of the lunar latitude. Therefore, the temperatures are always highest at lunar 
equator and lowest at the poles. The solar irradiance is inversely proportional to the square of the Moon-Sun distance $d$. Then, the lunar surface temperature $T$ is related to $d$ by

$$
T \infty d^{-\frac{1}{2}} \text {. }
$$

when other illumination conditions are the same. The seasonal oscillation of the Earth-Sun distance is expected to introduce seasonal oscillation to the lunar surface temperature. The maximum daytime lunar surface temperature is roughly $390 \mathrm{~K}$. Because the emissivity of the lunar surface is less than 1, the maximum measured BT will be less than the value.

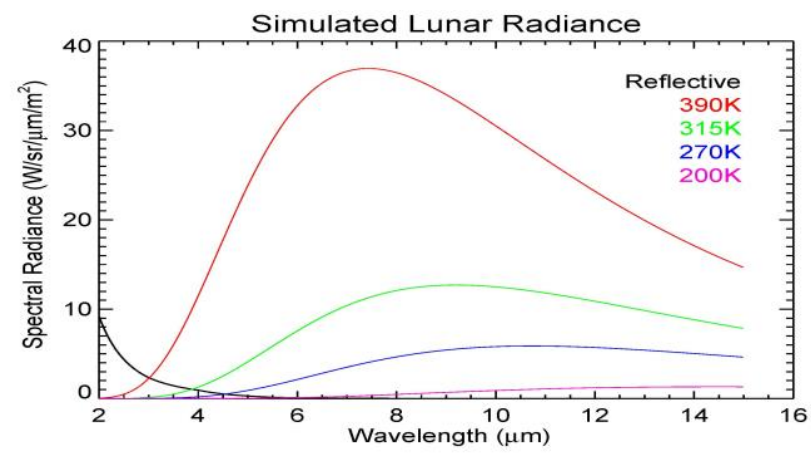

Figure 2. The simulated lunar spectral radiance components at different lunar surface temperatures.

The Moon has insufficient mass to retain atmosphere so the radiance $L$ from the lunar surface is observed by VIIRS directly without any atmospheric effect. It can be approximated as the sum of the reflected solar radiance and the thermal emission from the Moon ${ }^{10}$

$$
L(\lambda)=r \frac{\Omega L_{\text {solar }}}{\pi}+\varepsilon L_{\text {Moon }},
$$

where $r$ is the lunar directional reflectivity, $\Omega$ is the solid angle of the Sun viewed from the Earth or the Moon and $\varepsilon$ is the spectral emissivity of the lunar surface. At the infrared spectral range, the solar radiance $L_{\text {Solar }}$ can be modeled as the emission of a blackbody of $5900 \mathrm{~K}$. The lunar emissive radiance $L_{\text {Moon }}$ can be modeled as the emission of a blackbody of the lunar surface temperature. The surface temperature of the Moon varies considerably with location and solar illuminating condition. Depending on the local surface temperature, the amount of the contribution of the reflective and emissive lunar radiance is different. Figure 2 shows the thermal emission at different temperatures comparing with the reflected radiance. The emissivity of the lunar surface is less than 1 , so the thermal emission of the lunar surface should be less than the estimation. Detailed features in the lunar spectrum such as the Christiansen feature are omitted in this calculation ${ }^{11}$. Even so, the result still proves that the contribution of the reflective solar radiance is negligible beyond 6 $\mu \mathrm{m}$ when the surface temperatures are higher than $200 \mathrm{~K}$. It also suggests that to track the TEB performance, it is better to use lunar surface regions at higher temperature where the thermal contribution is more dominant.

Because the highest BT of the Moon still surpasses the dynamic range of all TEB detectors, the lunar images of all TEB detectors are partially saturated. The partial saturation of the lunar images can be clearly observed in Fig. 1. For the unsaturated pixels, their $d n$ are converted to radiance $L$ using Eqn. (1) with the detector gain coefficients calibrated onorbit and then their BT are calculated using Planck's equation.

\section{METHODOLOGY}

The idea of trending the radiometric calibration stability of thermal bands using lunar image has been developed for MODIS TEB calibration ${ }^{7}$. At nearly identical solar illumination conditions, the lunar surface temperatures are stable among events, after the compensation of the impact of the Moon-Sun distance variation to the surface temperatures disclosed in Eqn. (3). Then the long-term stability of the TEB calibration can be monitored by tracking the BT of the lunar surfaces. The trending must be based on the same regions on the Moon because of the significant spatial nonuniformity of the lunar surface BT. For VIIRS TEB, the difficulty is that all their detectors are partially saturated when viewing the Moon during the lunar calibration, although the saturation temperatures are band and detector dependent. 
We assume that the spatial variation of the lunar surface thermal map is the same for all these events. That is, the lunar pixels with BT of a certain percentile correspond to the same region of the Moon surface for all the observations. This assumption is supported by the histograms of the lunar surface BTs retrieved by VIIRS. As is shown in Fig. 3 for a few randomly selected events, the spatial distributions of the BT of the lunar surface almost repeat. Then if we track the hottest pixels after removing the same fraction of the lunar surfaces using an exclusion factor (defined below), the trended areas will be the same for all events. Here we use the same surface fraction instead of the same number of pixels because the size of the lunar images in pixels varies with the Moon-VIIRS distances $d_{\text {Moon_viirs. }}$.
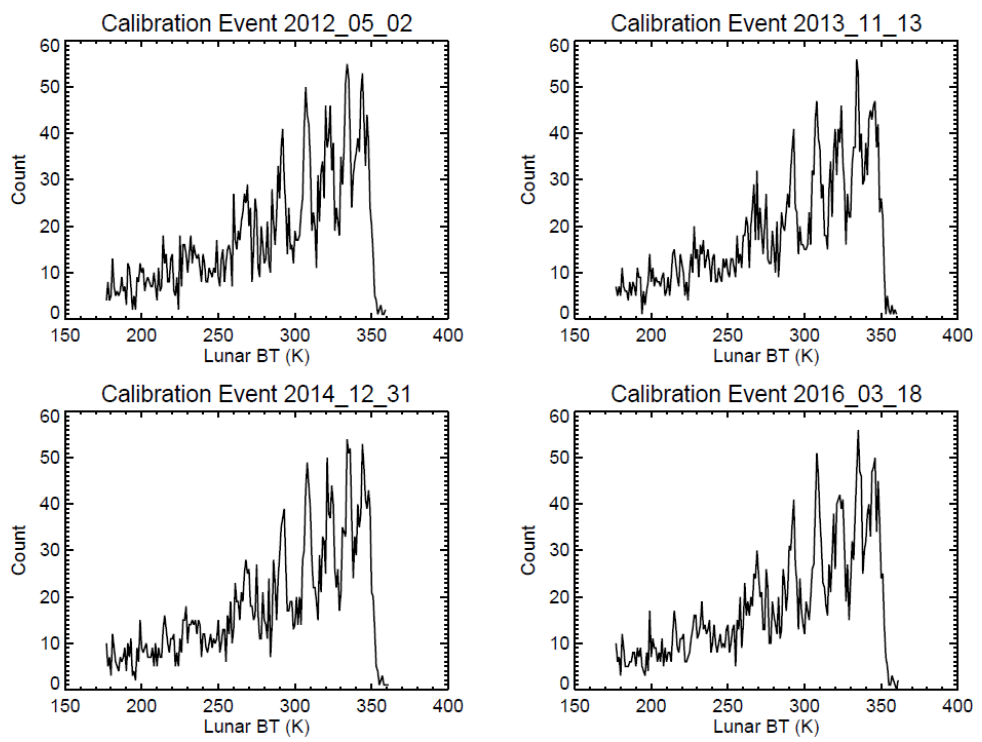

Figure 3. The histogram of the lunar BT retrieved using VIIRS calibration coefficients for a few randomly selected events.

An exclusion factor (mask) is defined on a detector basis to exclude the portion of the images that is potentially saturated for any lunar event. The size of the mask can be determined by the maximum limit of the saturated portion of the lunar images of all existing events. The fraction of the Moon illuminated at phase angle of 51 degrees is approximately $0.78^{12}$. We can calculate the number of M-band pixels being illuminated by

$$
N=\pi \cdot\left(\frac{r_{\text {Moon }}}{d_{\text {Moon }- \text { virrs }}} \cdot \frac{833}{0.742}\right)^{2} \cdot 0.78
$$

An alternative way is to directly count the lunar pixels above a certain radiance threshold from the lunar images. Both numbers are plotted in Fig. 4 for band I5 as an example. Different colors in (b) represent different detectors. We can see that the trends from the two approaches match each other. The counted numbers are a little greater than the estimated numbers because the peripheral pixels outside the boundary of the Moon are also illuminated as a result of the spatial spreading of the IFOV of detectors.

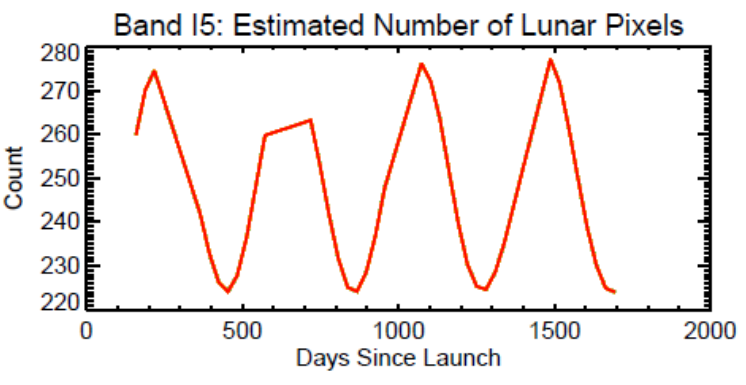

(a)

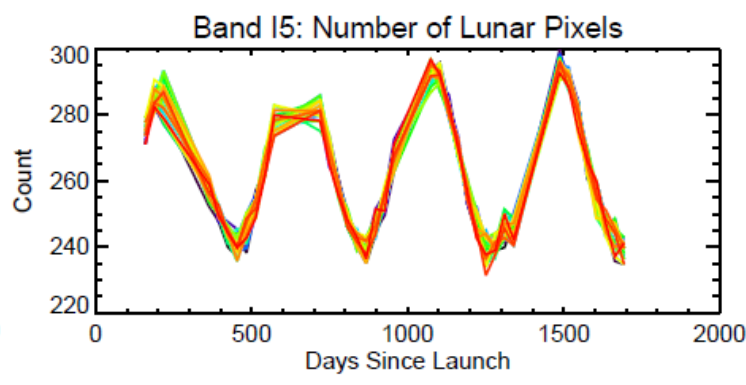

(b)

Figure 4. The number of lunar pixels (a) calculated from the illuminated portion of the Moon with Eqn. (5) or (b) counted from the lunar images based on radiance thresholds. 
The next step is to count the number of the saturated pixels $N_{\text {sat }}$ based on the raw DN without background correction. The data are 12 bit, so the saturated pixels all have a raw DN of 4095. The number of saturated pixels is shown for band I5 detectors in Fig. 5 (a). The number are detector dependent because the gains are detector dependent, as is shown in Fig. 5 (b). All these detectors cover nearly the entire lunar surface. Therefore, if the F-factor of a detector is high, or equivalently its gain is low, the detector's saturation is less significant or the number of the saturated signals during the lunar calibration is low. For all the single gain bands, the lunar pixels are aggregated every three pixels on-board. It is possible that an aggregated pixel has raw DN less than 4095 while one or two of the three un-aggregated pixels that compose the pixel is saturated. To identify these pixels, the data of band M13, which is not aggregated on-board, can be used as a bridge to approximately recover the raw DN of the un-aggregated pixels of other bands, assuming

$$
\frac{d n_{i, B}}{\sum_{i=1}^{3} d n_{i, B}}=\frac{d n_{i, M 13}}{\sum_{i=1}^{3} d n_{i, M 13}},
$$

for every aggregated pixel. An aggregated pixel is considered as saturated if one of its three un-aggregated components is saturated.

The fraction of the saturation is then calculated for each event by

$$
f=\frac{N_{s a t}}{N} \cdot \frac{1}{f_{o s}},
$$

where $f_{o s}$ is the oversampling factor being defined as the number of scans taken for the VIIRS swatch to move one detector IFOV in the along-track direction. The exclusion mask size is then set as the upper bound of these fractions with a margin currently set as 0.02 . Then, the number of pixels being excluded from the trending is calculated by the multiplication of the total pixels, the mask size and the oversampling factor. The trending of the saturation fraction, the size of the mask and the numbers of lunar pixels removed by the mask are plotted in Fig. 6.

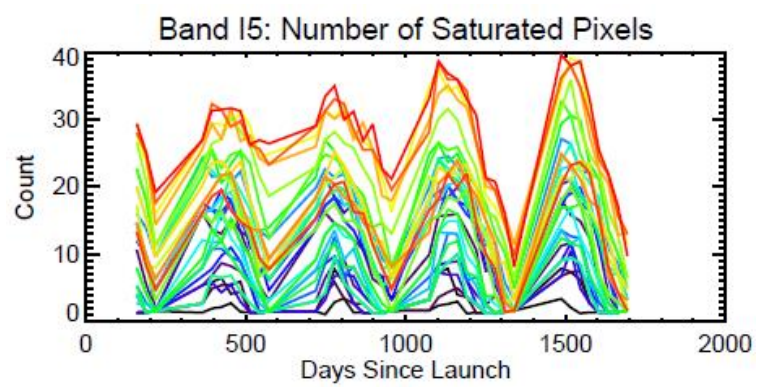

(a)

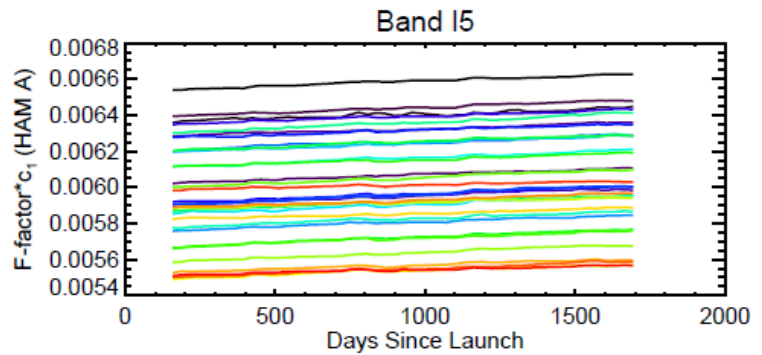

(b)

Figure 5. The trending of (a) the number of saturated lunar pixels and the F-factor (inverse of detector gains) for band I5.

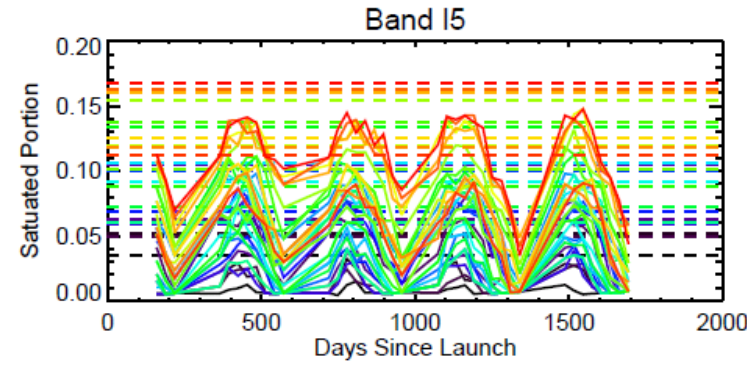

(a)

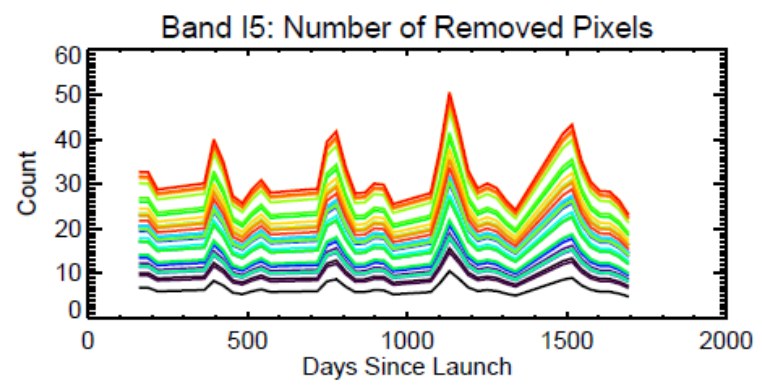

(b)

Figure 6. (a) The size of the mask (dashed line) is determined to be greater than the calculated saturation fraction (solid line) of all events and (b) The numbers of pixels excluded by the mask. 


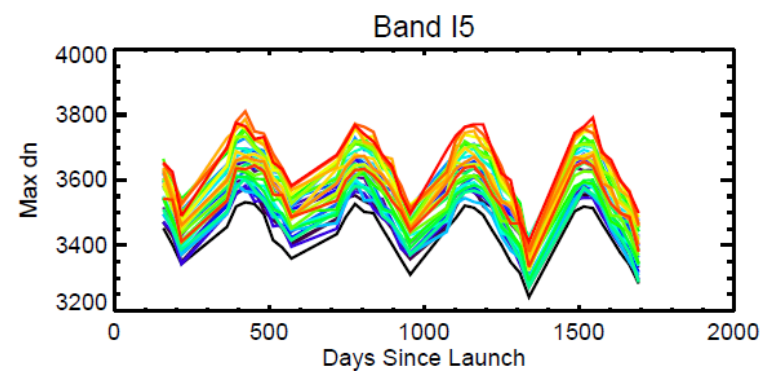

(a)

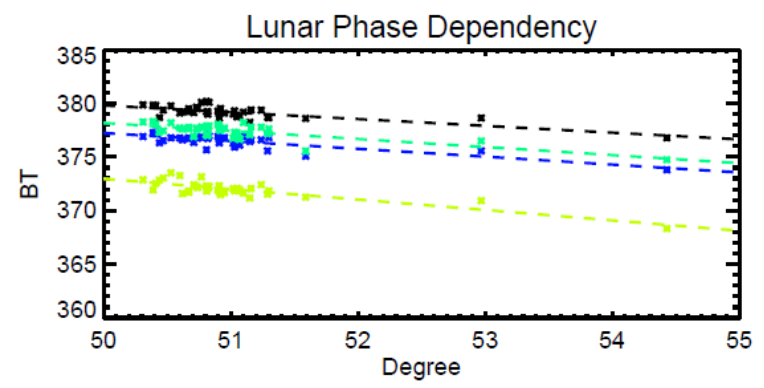

(c)

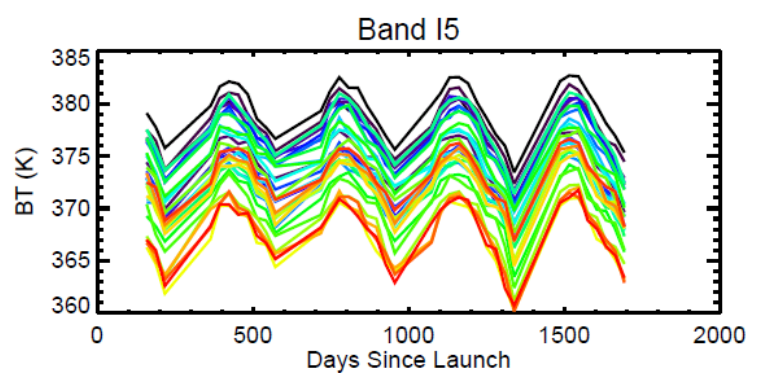

(b)

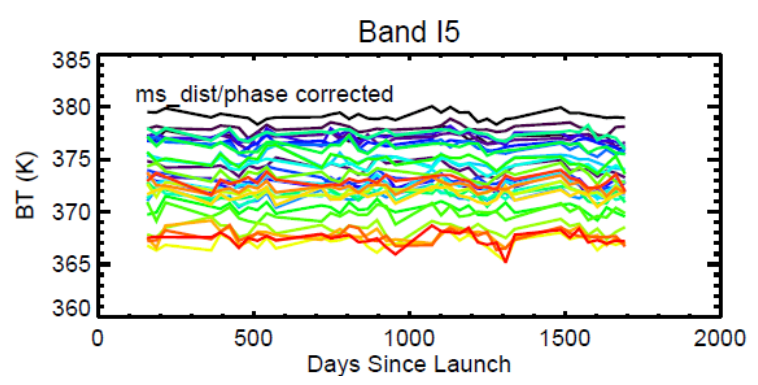

(d)

Figure 7. The trending of lunar $d n$, radiance and BT.

After excluding the pixels with the mask, the hottest remaining 6 pixels are trended, with the assumption that these pixels correspond to the same locations on the Moon. The background subtracted $d n$ and the retrieved BT are trended in the Fig. 7 (a) and (b), respectively, still using band I5 as an example. The seasonal oscillation of the BT is mostly due to the variation of the Moon-Sun distances that actually change the surface temperatures of the Moon. This can be compensated by Eqn. (3). The unit of $d_{\text {Moon_Sun }}$ is astronomical unit (AU). Therefore, the long-term average of the correction factor is 1 , and applying the correction does not change the long-term average of the retrieved BT.

Another factor that impacts the trending is the dependency of the BT on the lunar phase angles, as is shown in Fig. 7 (c) for a few detectors. A particular outlier in the data is corresponding to one calibration event that occurred on June $27^{\text {th }}$, 2015. Because of the constraint of the lunar orbit, the lunar phase angle at the time of observation is 54.43 degrees, significantly larger than others. As a result, the retrieved temperature for the event is about 2 degrees lower than the rest of the events. Because there is no existing lunar radiance model at thermal range similar to USGS ROLO irradiance model for reflective solar bands ${ }^{6}$, an empirical correction is applied by linear regression between the retrieved BT and the lunar phase angle. After the compensation of Moon-Sun distance and lunar phase, the lunar trending of band I5 are shown on a detector basis in Fig. 7 (d).

\section{RESULTS AND DISCUSSION}

\subsection{Surface BT trending}

The trending results of all other TEB, after the correction of Moon-Sun distance and lunar phase dependency, are provided in Fig. 8 on a detector basis. Band M13 is plotted before M12 because it is the only dual-gain band and the data are un-aggregated, which is different from M12, M14-16. The BT trending of detector 1 of band M12 is out of family because its detector gain is significantly higher than other detectors and it is saturated at higher BT. The drift of the BT through July, 2016, averaged for each band, is calculated through the linear fitting of the data and provided in Table 2. The band averaged standard deviation of the data is also tabulated. The numbers show that the BT has been stable overall within the $\pm 0.4 \mathrm{~K}$ range. The standard deviation of the data is a measure of the sensitivity of this monitoring approach.

The BT of all these tracked pixels is considerably higher than the 267 - $315 \mathrm{~K} \mathrm{BB}$ temperature range that is reached during BB WUCD calibration, as well as the BB operational temperature of $292.5 \mathrm{~K}$. In most of the cases, they are 
higher than the specified maximum detectable temperature $T_{\max }$ as is shown in Table 1 . The results confirm that the detector performance after calibration is still stable at the higher end of the dynamic temperature range.
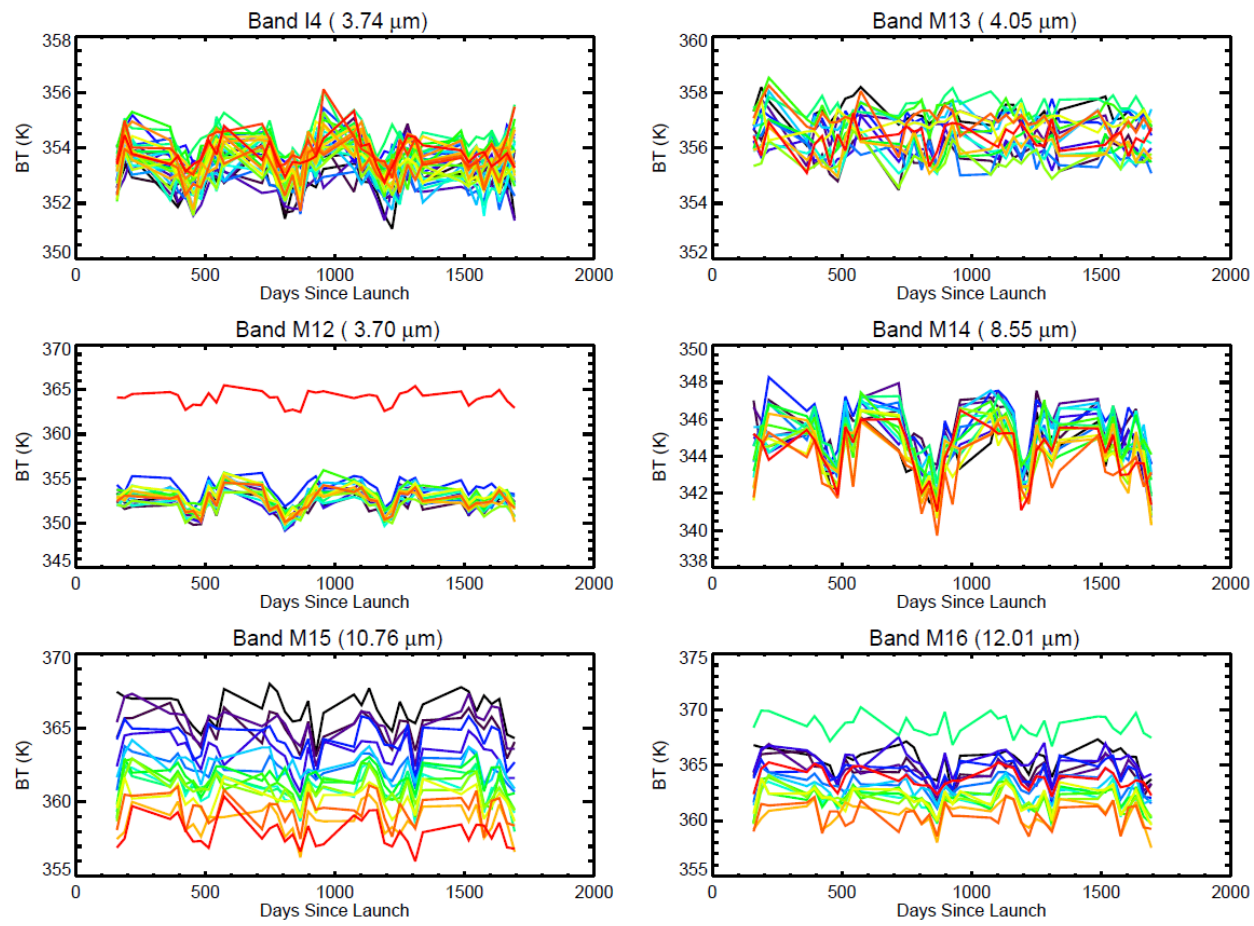

Figure 8. The detector-by-detector BT trending of the lunar surface for VIIRS TEB.

Table 2. The change of the BT of lunar surface 2011-2016.

\begin{tabular}{|c|c|c|}
\hline Band & BT Drift (K) & BT STD (K) \\
\hline I4 & -0.04 & 0.59 \\
\hline I5 & -0.17 & 0.52 \\
\hline M13 & -0.06 & 0.44 \\
\hline M12 & 0.07 & 1.09 \\
\hline M14 & -0.21 & 1.41 \\
\hline M15 & -0.30 & 1.01 \\
\hline M16 & -0.36 & 1.06 \\
\hline
\end{tabular}

\subsection{Inter-band BT comparison}

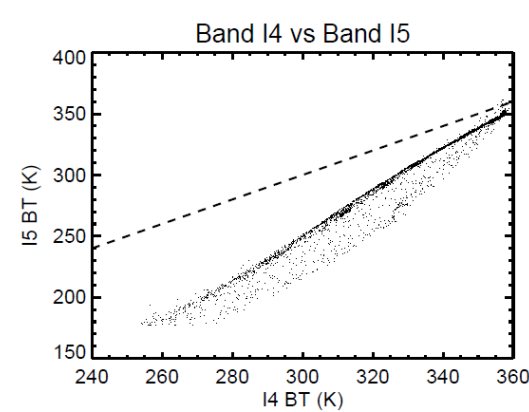

(a)

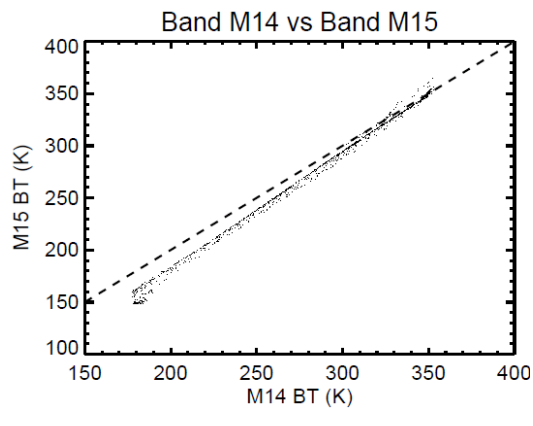

(b)

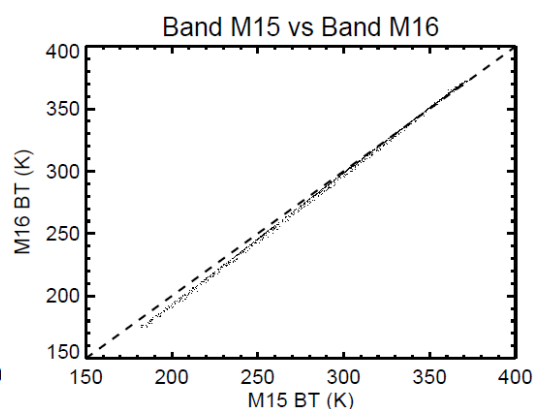

(c)

Figure 9. The relationship among the retrieved BT of different TEBs. The dashed line represents a reference line $\mathrm{x}=\mathrm{y}$ 
The scatter plots in Fig. 9 show the relationship among the retrieved BT of different bands for matching lunar pixels. The dashed line represents the reference line $\mathrm{x}=\mathrm{y}$. If these bands are perfectly registered to each other, the surface temperatures of the matching detectors should be identical. Therefore, the differences between the retrieved BT is mainly due to the emissivity difference at different wavelength since $T_{b}=\varepsilon T$ under Rayleigh-Jeans law. In Fig. 9 (a), the scattering of the data are significantly below the reference line, indicating the emissivity of band I4 at $3.74 \mu \mathrm{m}$ is significantly greater than the emissivity of band I5 at $11.45 \mu \mathrm{m}$. In Fig. 9 (b), the emissivity of band M14 at $8.55 \mu \mathrm{m}$ is also greater than the emissivity of band M15 at $10.76 \mu \mathrm{m}$. In Fig. 9 (c), the emissivity of band M15 and band M16 at $12.01 \mu \mathrm{m}$ are similar. The slope of the curve is a measure of the emissivity ratio. The deviation of the data from a straight line is probably because the Rayleigh-Jeans law is just an approximation of blackbody radiation below $6 \mu \mathrm{m}$, so the approximation $T_{b}=\varepsilon T$ is not accurate for some of the TEBs.

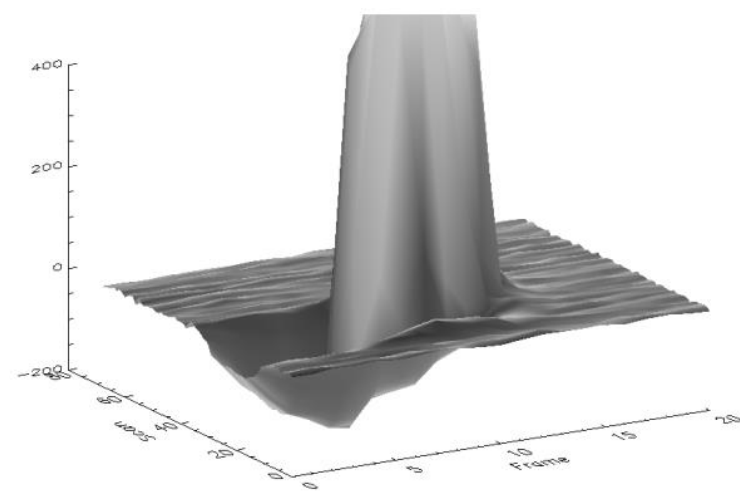

(a)

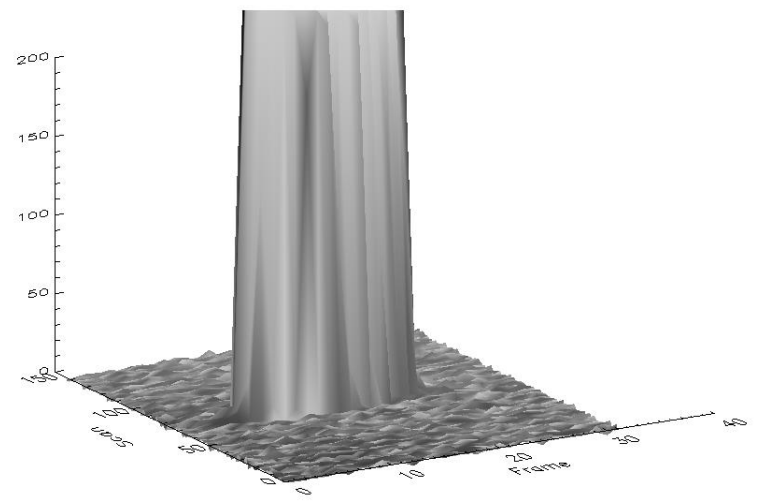

(b)

Figure 10. The lunar images of (a) Terra MODIS band 27 detector 8 that is contaminated by electric crosstalk and (b) VIIRS band $\mathrm{I} 4$ detector 1 that is clear of crosstalk.

\subsection{Crosstalk Examination}

The lunar images of thermal bands are good indicator of signal contamination among bands or crosstalk ${ }^{13}$. Fig. 10 (a) shows the lunar images of Terra MODIS band 27 detector 8. Because of the electric crosstalk from other bands, the lunar images show tails at the peripheral region of the Moon. The lunar image of VIIRS band I4 detector 1 shown in Fig. 10 (b) has no tail and indicates the detector is clear of crosstalk. All VIIRS lunar TEB images have been constantly monitored. As of now, there is no indication of the existence of crosstalk.

\section{CONCLUSION}

The long-term stability of the VIIRS TEB is monitored using lunar observations. With the on-orbit BB calibrated coefficients, the BT of the lunar surfaces can be retrieved and trended. The results presented in the paper show that these bands have been radiometrically stable throughout VIIRS lifetime for al TEB detectors, within an uncertainty level of $\pm 0.4 \mathrm{~K}$. The results also suggest that the Moon can be used for the on-orbit characterization of the TEB for remote sensing instruments with regular lunar observation capability. The monitoring will be performed continuously, as part of the on-orbit calibration effort. Further improvement of the methodology may reference temperature map generated by other lunar exploration sensors such as diviner aboard NASA's lunar reconnaissance orbiter.

\section{REFERENCES}

[1] Mills, S., "VIIRS radiometric calibration algorithm theoretical basis document ATBD," Northrop Grumman, Doc. No.: D43777 (2010).

[2] Guenther, B., Barnes, W. L., and Murphy, R. E., "A calibration algorithm design and analysis for VIIRS thermal emissive bands based on the EOS MODIS approach," IGARSS Proc., 5, 3036-3038 (2003). 
[3] Xiong, X., Butler, J., Chiang, K., Efremova, B., Fulbright, J., Lei, N., McIntire, J., Oudrari, H., Sun, J.,Wang, Z., and Wu, A., "VIIRS on-orbit calibration methodology and performance." J. Geophys. Res. Atmos. 119, 5065-5078 (2014).

[4] Cao, C., De Luccia, F., Xiong, X., Wolfe, R., and Weng, F. "Early on-orbit performance of the Visible Infrared Imaging Radiometer Suite (VIIRS) onboard the Suomi National Polar-Orbiting Partnership (S-NPP) satellite.” IEEE Trans. Geosci. Remote Sens., 52, 1142-1156. (2014).

[5] Hass, E., Moyer D., Moy, and G., De Luccia, F., "SNPP VIIRS thermal emissive band performance after three years on-orbit," IGARSS, Proc., 3675-3678 (2015).

[6] Kieffer, H. H., and Stone, T. C., "The spectral irradiance of the Moon,” Astronom. J. 129, 2887-2901 (2005).

[7] Wang, Z., Xiong, X., Chen, H., and Madhavan, S., "On-orbit radiometric stability assessment of MODIS thermal emissive bands with lunar observation,” Proc. SPIE 8866, 886604 (2013).

[8] Sun, J., Xiong, X., and Butler, J., "NPP VIIRS on-orbit calibration and characterization using the Moon," Proc. SPIE 8510, 851054 (2012).

[9] Wang, Z., Xiong, X., and Li, Y., "Improved band-to-band registration characterization for VIIRS reflective solar bands based on Lunar observations," Remote Sens., 8(1), 27 (2016).

[10] Shaw, J. A., "Modeling infrared lunar radiance," Opt. Eng. 38(10), 1763-1764 (1999).

[11] Paige, D. A., et al. "The lunar reconnaissance orbiter diviner lunar radiometer experieent," Space Sci. Rev., 150, 125-160 (2010).

[12] U. S. Navy observatory website, http://aa.usno.navy.mil/data/docs/MoonFraction.php

[13] Sun, J., Madhavan, S., Wenny, B. N., and Xiong, X., "Terra MODIS band 27 electronic crosstalk: Cause, impact, and mitigation," Proc. SPIE 8176, 81760Z (2011). 